\title{
Disruption of Fixation Reveals Latent Sensorimotor Processes in the Superior Colliculus
}

\author{
너day K. Jagadisan ${ }^{1,4}$ and $@$ Neeraj J. Gandhi ${ }^{1,2,3,4}$ \\ ${ }^{1}$ Department of Bioengineering, ${ }^{2}$ Department of Otolaryngology, ${ }^{3}$ Department of Neuroscience, and ${ }^{4}$ Center for the Neural Basis of Cognition, University of \\ Pittsburgh, Pittsburgh, Pennsylvania 15213
}

\begin{abstract}
Executive control of voluntary movements is a hallmark of the mammalian brain. In the gaze-control network, this function is thought to be mediated by a critical balance between neurons responsible for generating movements and those responsible for fixating or suppressing movements, but the nature of this balance between the relevant elements-saccade-generating and fixation-related neuronsremains unclear. Specifically, it has been debated whether the two functions are necessarily coupled (i.e., push-and-pull) or independently controlled. Here we show that behavioral perturbation of ongoing fixation with the trigeminal blink reflex in monkeys (Macaca mulatta) alters the effective balance between fixation and saccade-generating neurons in the superior colliculus (SC) and can lead to premature gaze shifts reminiscent of compromised inhibitory control. The shift in balance is primarily driven by an increase in the activity of visuomovement neurons in the caudal SC, and the extent to which fixation-related neurons in the rostral SC play a role seems to be linked to the animal's propensity to make microsaccades. The perturbation also reveals a hitherto unknown feature of sensorimotor integration: the presence of a hidden visual response in canonical movement neurons. These findings offer new insights into the latent functional interactions, or lack thereof, between components of the gaze-control network, suggesting that the perturbation technique used here may prove to be a useful tool for probing the neural mechanisms of movement generation in executive function and dysfunction.
\end{abstract}

Key words: fixation; impulsivity; microsaccades; sensorimotor integration; superior colliculus; visuomovement neurons

\section{Significance Statement}

Eye movements are an integral part of how we explore the environment. Although we know a great deal about where sensorimotor transformations leading to saccadic eye movements are implemented in the brain, less is known about the functional interactions between neurons that maintain gaze fixation and neurons that program saccades. In this study, we used a novel approach to study these interactions. By transient disruption of fixation, we found that activity of saccade-generating neurons can increase independently of modulation in fixation-related neurons, which may occasionally lead to premature movements mimicking lack of impulse control. Our findings support the notion of a common pathway for sensory and movement processing and suggest that impulsive movements arise when sensory processes become "motorized."

\section{Introduction}

Active vision entails interacting with the environment through alternating patterns of fixations and redirections of gaze or saccades. The behavioral transition between fixation and saccade generation is mediated by a neuronal shift between movementsuppressing neurons and movement-generating neurons in the

\footnotetext{
Received 0ct. 6, 2015; revised April 12, 2016; accepted April 12, 2016.

Author contributions: U.K.J. and N.J.G. designed research; U.K.J. performed research; U.K.J. analyzed data; U.K.J. and N.J.G. wrote the paper.

This work was supported by National Institutes of Health Grant EY022854 to N.J.G. We thank Ivan Smalianchuk for useful discussions during preparation of the manuscript.

The authors declare no competing financial interests.

Correspondence should be addressed to Uday K. Jagadisan, 153 Eye and Ear Institute, 203 Lothrop Street, Pittsburgh, PA 15213. E-mail: kj.udayakiran@gmail.com.

DOI:10.1523/JNEUROSCI.3685-15.2016

Copyright $\odot 2016$ the authors $\quad 0270-6474 / 16 / 366129-12 \$ 15.00 / 0$
}

oculomotor neuraxis. The superior colliculus (SC), which is a major node in the gaze-control pathway (Gandhi and Katnani, 2011), is ideally situated to implement the transition. Movement suppression is controlled by so-called "fixation-related neurons" in the rostral SC (Munoz and Wurtz, 1993a). These neurons are active during fixation, which is likely maintained by preparatory activity associated with programming of microsaccades (Hafed et al., 2009). Crucially, they suppress their discharge before large saccades. Movement generation is mediated by visuomovement neurons in the caudal SC (Sparks and Mays, 1990; Dorris et al., 1997) that burst during saccades. Intriguingly, these neurons also burst in response to a visual stimulus.

One line of evidence points to a reciprocal relationship between the rostral and the caudal SC in maintaining the balance between fixation and saccade generation under normal conditions. Inactivation of the rostral SC leads to uninhibited saccade 
generation to peripheral visual targets (Munoz and Wurtz, 1993b) or stable offsets during fixation (Hafed et al., 2008; Goffart et al., 2012). Conversely, microstimulation in the caudal SC generates saccades (Robinson, 1972), and microstimulation of the rostral SC interrupts ongoing large movements (Munoz et al., 1996), perhaps through interference induced by stimulationevoked small-amplitude saccades (Gandhi and Keller, 1999). However, the inability to record neural activity simultaneously during these manipulations makes it difficult to gauge the functional relationship between fixation and saccade-generating mechanisms, and whether the interactions extend beyond movement generation to other features of the sensorimotor transformation. For instance, does the state of the network during fixation influence the visual response of visuomovement neurons in caudal SC? Are activation changes in one network obligatorily linked to changes in the other, and how do these changes influence fixation, microsaccade, and saccade behavior? It is important to mention here that we use the term "network" in this paper to broadly refer to a collection of putatively interconnected neurons thought to perform a specified function.

We used an alternative approach-naturalistic, noninvasive, and transient behavioral perturbation of fixation-that permitted simultaneous recording of neural activity. We reasoned that if fixation is behaviorally disrupted, it must affect activity in the fixation-related network, altering the balance between the fixation and saccade-generating networks. Hence, on some trials within a session, we presented an air puff to the subject's eye to evoke the trigeminal blink reflex (Berardelli et al., 1985) during initial fixation of a central target. Blinks disrupt fixation by transiently removing foveal visual input and producing an associated blink-related eye movement (Rottach et al., 1998; Gandhi and Bonadonna, 2005), during which activity in the pontine omnipause neurons, a low-level node in the fixation network, is suppressed (Schultz et al., 2010). We asked whether and how this perturbation affects the evolution of activity in the fixationrelated and saccade-generating networks in the SC. Note that the blink was induced while the animal directed its gaze to a central target and, crucially, fixation of this target was re-established before a peripheral stimulus was illuminated. Thus, we were able to circumvent potential confounds of transient changes caused by the blink perturbation. In a small percentage of trials, blinks biased the network-level balance toward caudal SC neurons sufficiently to generate premature saccades. More importantly, we observed changes in network activity even when saccades were not triggered prematurely. The stimulus-evoked visual response of visuomovement neurons was enhanced and, surprisingly, putative movement neurons also exhibited a visual response. Blinkinduced modulation of rostral SC activity, which was more closely associated with the animal's propensity to make microsaccades, did not obey the purported coupled relationship with saccade-generating neurons in caudal SC. These results have a potentially deep impact on our understanding of mechanisms mediating voluntary control of gaze and its dysfunction in neuropsychiatric disorders.

\section{Materials and Methods}

General and surgical procedures. All experimental and surgical procedures were approved by the Institutional Animal Care and Use Committee at the University of Pittsburgh and were in compliance with the U.S. Public Health Service policy on the humane care and use of laboratory animals. We used three adult rhesus monkeys (Macaca mulatta; two male, ages 8 and 6 years; one female, age 10 years) for our experiments. One animal died during the course of the study, so we completed the remaining experiments with the third animal. Under isoflurane anesthesia, a craniotomy that allowed access to the SC was performed on each animal and a recording chamber was secured to the skull over the craniotomy. In addition, posts for head restraint and scleral search coils to track gaze shifts were implanted. After recovery, each animal was trained to perform standard eye-movement tasks for a liquid reward.

Visual stimuli and behavior. Visual stimuli were displayed either by back projection onto a hemispherical dome (Monkeys BB and WM) or on a LED-backlit flat screen television (Monkeys BB and BL). Stimuli were white squares on a dark gray background, $4 \times 4$ pixels in size, and subtended $\sim 0.5^{\circ}$ of visual angle. Eye position was recorded using the scleral search coil technique, sampled at $1 \mathrm{kHz}$. Stimulus presentation and the animal's behavior were under real-time control with a LabVIEW-based controller interface (Bryant and Gandhi, 2005). After initial training and acclimatization, the monkeys were trained to perform a delayed saccade task. Each subject was required to initiate the trial by acquiring fixation on a central fixation target. Next, a target appeared in the periphery, but the fixation point remained illuminated for a variable $500-1200 \mathrm{~ms}$, and the animal was required to delay saccade onset until the fixation point was extinguished (GO cue). Trials in which fixation was broken before peripheral target onset were removed from further analyses.

Perturbations. On a small percentage of trials ( $\sim 15-20 \%)$, fixation was perturbed by delivering an air puff to the animal's eye to invoke the trigeminal blink reflex. Compressed air was fed through a pressure valve and air flow was monitored with a flow meter. To record blinks, we taped a small Teflon-coated stainless-steel coil (similar to the ones used for eye tracking, but smaller in coil diameter) to the top of the eyelid. The air pressure was titrated during each session to evoke a single blink (typically 15-30 psi). Trials in which the animal blinked excessively or did not blink were aborted and/or excluded from further analyses. Air-puff delivery was timed to evoke blinks $400-100$ ms before target onset. Blinks typically lasted 50-100 ms, and we removed trials in which fixation of the central target was not re-established before a stimulus was illuminated in the periphery to ensure that vision of fixation spot or the incoming stimulus was not altered at the time of its onset. For two of the monkeys (BB and $\mathrm{BL}$ ), we also used two other forms of perturbation. First, the central fixation target was transiently blanked out for 50-100 ms at approximately the same times that a blink would have occurred on blink trials (before peripheral target appearance). We refer to these as targetblank trials. In other experiments, an air puff was introduced to the animal's ear. Trials in which the animal blinked defensively in response to the ear puff were removed from further analysis. The animals typically adapted to this perturbation after a few trials.

Electrophysiology. During each recording session, a tungsten microelectrode was lowered into the SC chamber using a hydraulic microdrive. Neural activity was amplified and bandpass filtered between $200 \mathrm{~Hz}$ and $5 \mathrm{kHz}$ and fed to a digital oscilloscope for visualization and spike discrimination. A window discriminator was used to threshold and trigger spikes on-line, and the corresponding spike times were recorded. The location of the electrode in the SC was confirmed by the presence of visual and movement-related activity as well as the ability to evoke fixed-vector saccadic eye movements at low stimulation currents $(20-40 \mu \mathrm{A}, 400 \mathrm{~Hz}$, $100 \mathrm{~ms}$ ). Before beginning data collection for a given neuron, its response field was estimated. During data collection, the saccade target was placed either in the neuron's response field or at the diametrically opposite location (reflected across both axes) in a randomly interleaved manner. For recordings in the rostral SC, stimuli were presented at one of two or four locations at an eccentricity sufficient to induce a reduction in activity during the large-amplitude saccade. In two of the monkeys (BB and $\mathrm{BL})$, we also performed recordings with a 16-channel linear microelectrode array (Alpha Omega Engineering; $150 \mu \mathrm{m}$ spacing between electrode contacts). Neural activity was digitized, filtered, and recorded with the Grapevine Scout system (Ripple). Since the electrode's approach was normal to the SC, neurons recorded with the array typically had comparable response fields.

Data analysis and preprocessing. Data were analyzed using a combination of in-house software and Matlab. Eye-position signals were smoothed with a phase-neutral filter and differentiated to obtain velocity 
traces. Saccades were detected using standard velocity criteria. The animal was considered to be maintaining fixation if the gaze remained within a $2-3^{\circ}$ window around the fixation target. Slow, blink-related eye movements were detected using lower-velocity criteria. We also detected microsaccades that occurred during a trial by using a velocity criterion based on the overall variability in the velocity signal for that trial. The adaptive algorithm enabled us to account for changes in fixation patterns and movements over time for each subject. We visually verified that microsaccades were being detected accurately.

Raw spike density waveforms were computed for each neuron and each trial by convolving the spike trains with a Gaussian kernel (width: 4 $\mathrm{ms}$ for the caudal SC, $10 \mathrm{~ms}$ for the rostral SC). For a given neuron and target location, spike densities were averaged across trials after eventrelated alignment (perturbation/target/saccade onset). Caudal SC neurons were classified as task-related (visual and/or movement) if the activity during the visual epoch (100-250 ms following target onset) and/or the premotor epoch ( -100 to $50 \mathrm{~ms}$ around saccade onset) was significantly elevated above baseline. Rostral SC neurons were classified as fixation-related if their activity during the premotor epoch of large saccades was significantly reduced below baseline. We used a firing-rate criterion to increase confidence that we were using only meaningful neurons in our analysis. This was especially important for the multichannel recordings as almost all channels had some activity due to crossover spikes or noise. Hence, we only considered caudal SC neurons that had a maximum firing rate of $\geq 100$ spikes/s and rostral SC neurons that had a tonic baseline firing rate of $\geq 20$ spikes/s. Further, we only used neurons that had $\geq 5$ trials for a given condition (e.g., early saccades following blink perturbation). Since the animals rarely made errors in the control condition, we did not have a sufficient number of neurons that met this criterion for that condition to warrant further analysis. To enable comparison of spike densities during the perturbation epoch, the perturbation-related alignment for no-perturbation trials was performed by random assignment of "perturbation" times from the distribution of times from perturbation trials for that session. We also normalized the trial-averaged spike density of each neuron to enable meaningful averaging across the population. The activity of each caudal $\mathrm{SC}$ neuron was normalized by its peak firing rate across conditions. The activity of each rostral SC neuron was normalized by the baseline firing rate across conditions.

Statistical comparisons of neural activity between conditions were performed using nonparametric tests (Wilcoxon rank-sum and signed-rank tests). Activity comparisons used either the average activity in a window or the instantaneous activity (sliding $1 \mathrm{~ms}$ bin), as appropriate. Where applicable, multiple comparisons were corrected for Bonferroni correction. A $p$ value $<0.01$ was used to assess statistical significance, unless otherwise specified.

To quantify the functional properties of caudal SC neurons as visual, visuomovement, or movement-related, we computed the visuomovement index (VMI) for each neuron. VMI was calculated as $V M I=\frac{M-V}{M+V}$, where $V$ is the baseline-subtracted activity during the visual epoch, and $M$ is the delay period-subtracted activity during the saccade epoch. We also calculated a modulation index to quantify the perturbation-induced modulation in the neurons' visuomovement activity. The modulation index was calculated for the visual epoch as $\frac{V_{p}-V_{c}}{V_{p}+V_{c}}$, where $V_{p}$ and $V_{c}$ are the baselinesubtracted activity during the visual epoch in perturbation and control trials, respectively.

\section{Results}

We perturbed fixation by inducing a blink and recorded neural activity from the SC in three monkeys performing a delayedresponse task (Fig. 1a). During the control condition, the animals correctly initiated the eye movement after fixation point offset on 98.0\% percent of the trials. The black trace (Fig. 1a, Eye) illustrates the temporal profile of a typical saccade and the black histogram (Fig. 1b) shows the distribution of reaction times (mean $\pm \mathrm{SD}, 276 \pm 46 \mathrm{~ms}$ ). On perturbation trials, the blink was

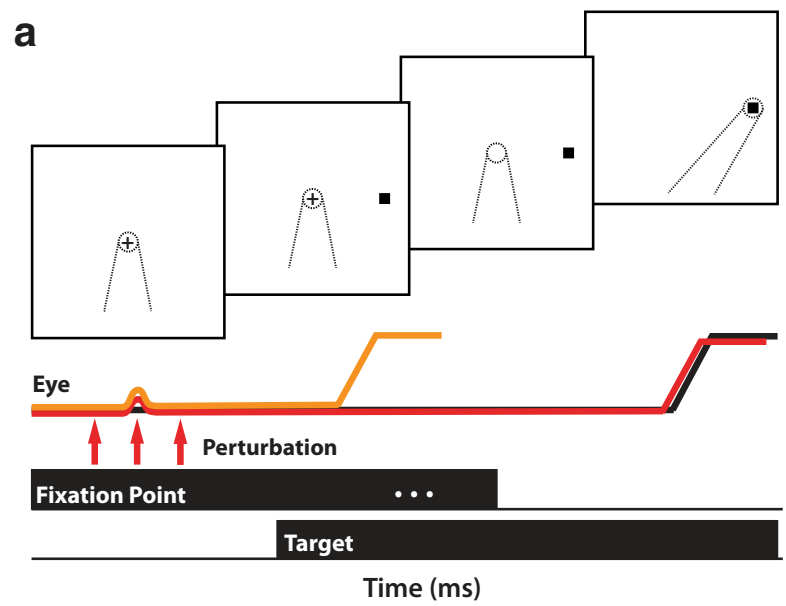

b

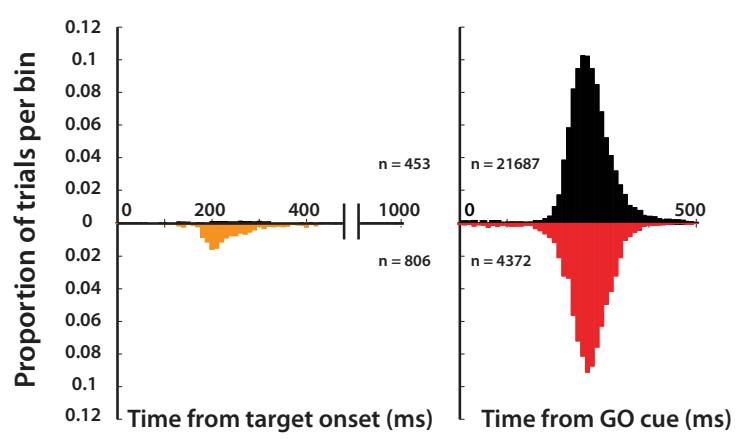

C

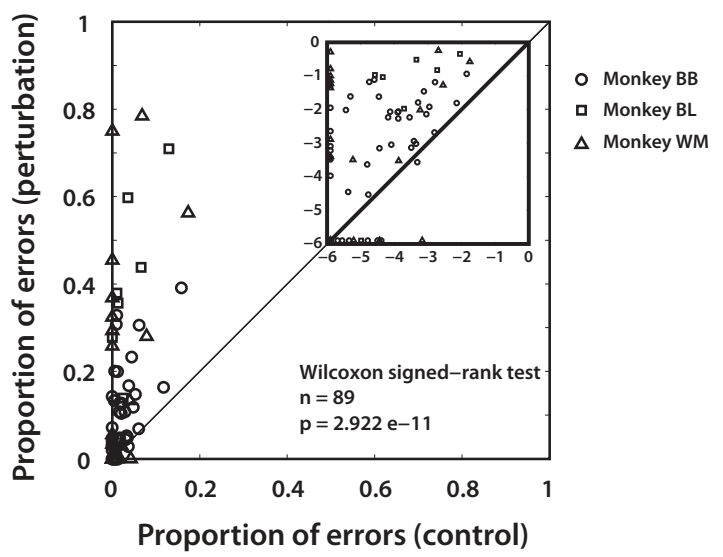

Figure 1. Behavior in control and perturbation conditions. $\boldsymbol{a}$, Schematic of the delayedsaccade task. The red arrows indicate that blink-perturbation times preceded target appearance. The temporal traces in the bottom rows are schematics of typical eye-position profiles in the absence of perturbation (control, black) and with perturbation leading to premature (gold) and regular latency saccades (red). Radial position is plotted. Hence all deflections are positive. $\boldsymbol{b}$, Histograms for the distribution of saccade reaction times with regular (right) and early (left) latencies for control (top) and perturbation (bottom) trials. The offset of fixation point represents the $\mathrm{G} 0$ cue. c, Proportion of errors in the perturbation condition (ordinate) plotted against the control condition (abscissa). Each point represents a session. Symbols represent individual subjects. The unity line is on the diagonal. The inset replots the same data on log-log axes for better visualization.

always induced during the initial fixation period (Fig. $1 a$, small bumps in the colored eye-position traces above the red arrows). We only analyzed trials in which the blink had completed and fixation re-established before a target was illuminated in the visual periphery. For the majority of trials (4372 of 5178; 84.4\%), the animals produced a saccade after the saccade-initiation cue, much like the behavior seen during control trials. The red trace (Fig. 1a) represents a typical example and the same color histo- 
a
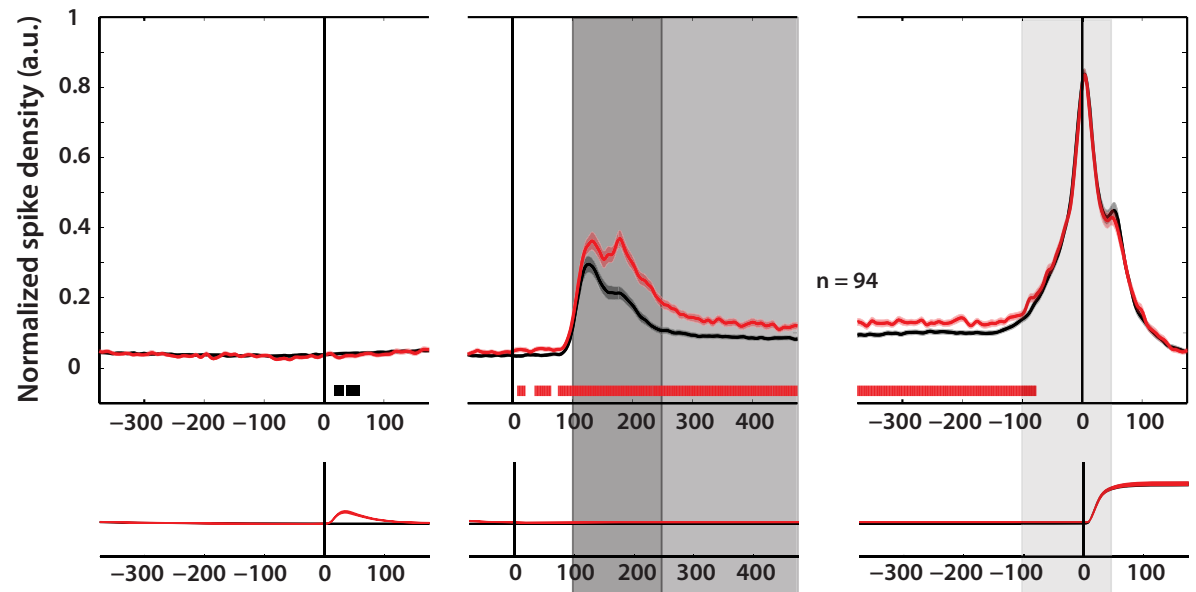

Time from blink onset (ms) Time from target onset (ms) Time from saccade onset (ms)

b

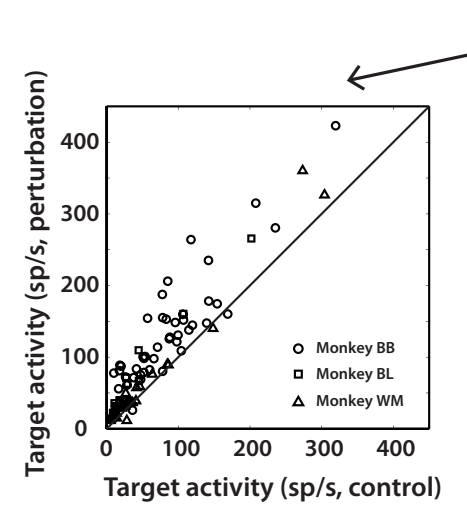

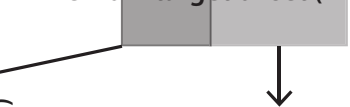

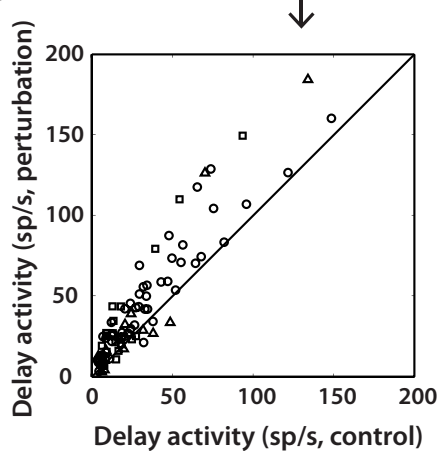

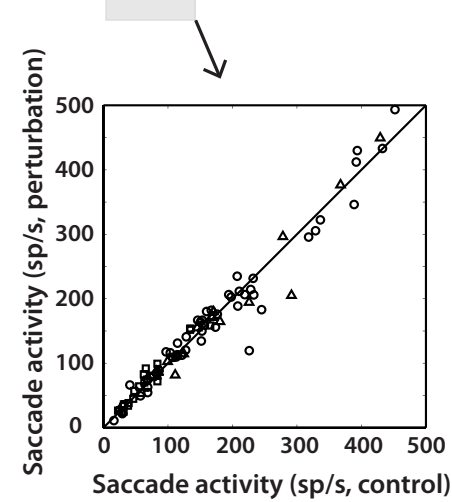

Figure 2. Population activity in the caudal SC. $\boldsymbol{a}$, Population average (mean \pm SEM) of caudal SC neurons (normalized) for the control (black) and perturbation (red) conditions. The tick marks near the base of the figure indicate time points at which the difference between the two conditions was significantly different (Wilcoxon signed-rank test, $p<0.01$ ). The color of the tick mark indicates the condition in which activity was higher. The bottom row shows mean eye-position traces in the two conditions. $\boldsymbol{b}$, Scatter plots of individual neuron activities $(n=94)$ during the target-evoked response period (left; $\boldsymbol{a}$, dark-shaded epoch), delay period (middle; $\boldsymbol{a}$, intermediate-shaded epoch), and saccade period (right; $\boldsymbol{a}$, light-shaded epoch) in the perturbation condition plotted against the control condition. Unity line is on the diagonal. Each symbol corresponds to a different monkey. Activity was enhanced during the target and delay epochs but not during the saccade epoch for a majority of neurons.

gram (Fig. $1 b$ ) shows the associated latency distribution (mean \pm $\mathrm{SD}, 271 \pm 50 \mathrm{~ms}$ ). On a smaller subset of trials (806 of 5178; $15.6 \%$ ), a saccade was produced prematurely during the delay period. The gold trace (Fig. 1a) denotes a typical example and the same color histogram (Fig. $1 b$ ) displays the latency distribution. The increase in likelihood of premature saccades was observed not just across the entire dataset but also during a majority of sessions in every animal (Fig. $1 c$; Wilcoxon signed-rank test, $n=$ $89, p<0.0001)$. Neural activity recorded during blink trials that did not alter behavioral performance ("red" data) provides an opportunity to assess the latent functional interactions between fixation and saccade-generating networks without introducing any confounds from activity associated with saccade execution. Analyses of these data constitute the major focus of this study. Discharge patterns associated with the smaller subset of blink data that yielded premature saccades ("gold" data) are considered briefly at the end of the Results section.

\section{Subthreshold enhancement of stimulus-evoked activity following disruption of fixation}

Figure $2 a$ shows the population activity of 94 caudal SC neurons recorded in the intermediate layers during control (black) and perturbation (red) trials with regular latency saccades. The visual response was strongly enhanced at the population level (Wil- coxon signed-rank test, $p<0.01$ ), as indicated by the significance bands along the bottom of the figure. Note that the perturbation occurred before target onset and the eyes were stationary and refixated on the fixation point at the time of the visual burst (Fig. $2 a$; bottom row shows mean eye-position traces for each condition). Activity was also enhanced significantly into the delay period. Scatter plots in Figure $2 b$ illustrate that the increase was observed regardless of the neurons' firing rate and, in several cases, the difference between conditions was of the order of $\geq 100$ spikes/s. This suggests that the perturbation-induced changes to the saccade-generating circuitry were strong and long-lasting, persisting several hundreds of milliseconds into the trial (left and middle panels). In contrast, the activity during the motor burst was comparable between control and perturbation conditions (Fig. 2b, right).

\section{A hidden visual response is present in putative movement neurons}

Next, we studied how the disruption of fixation affected the functional properties of these neurons. The VMI (see Materials and Methods), a measure of the relative strengths of a neuron's visual and saccade-related bursts, has previously been used to sort SC and frontal eye field neurons into functional classes (Shen and Paré, 2007; Cohen et al., 2009). Negative values (closer to -1) 


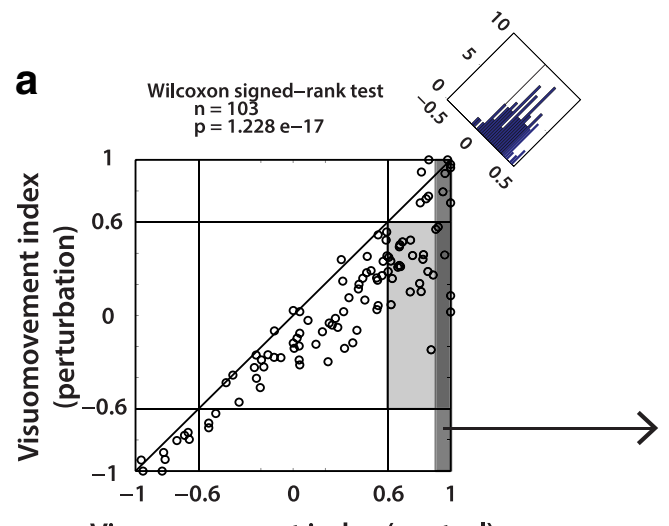

Visuomovement index (control)

\section{C}

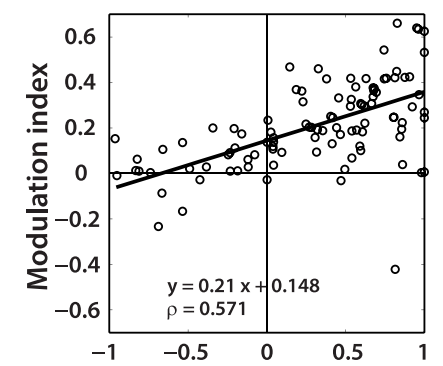

Visuomovement index (control) b
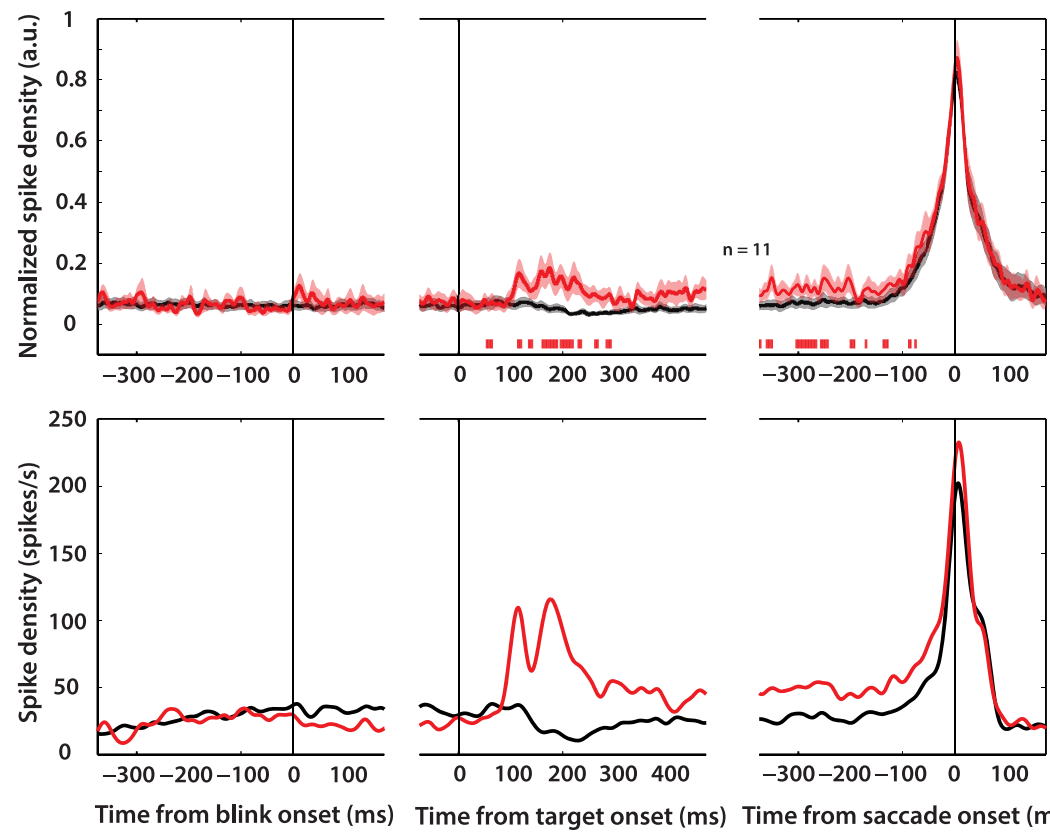

$-300-200-100 \quad 0 \quad 100$

Time from target onset (ms) Time from saccade onset (ms)

Figure 3. Latent visual response in putative movement neurons. $\boldsymbol{a}$, VMI (see Materials and Methods) calculated from perturbation trials plotted against VMI from control trials. VMI significantly decreased in perturbation trials (neurons became more "visual"). Note that neurons in the light-shaded rectangle that would typically be classified as "movement" neurons based on activity in the control condition (VMI, >0.6) become visuomovement neurons under perturbation. The histogram shows the distribution of VMI differences between the two conditions. $\boldsymbol{b}$, Top, Population activity of 11 movement neurons, defined as those with VMI $>0.9$ ( $\boldsymbol{a}$, dark-shaded region), in control and perturbation conditions. Bottom, Activity of an exemplar movement neuron showing strong unmasking of a latent visual response following the perturbation. $c$, Modulation index in perturbation trials relative to control trials plotted as a function of VMl in control trials. The correlation was significantly positive, i.e., more movement-like cells were more likely to be modulated by the perturbation during the visual epoch.

indicate that the neuron is primarily visually driven and positive values (close to +1 ) indicate that the neuron is primarily movement-related, with values in between representing a combination of the two processes. We computed the VMI for each neuron based on its activity on control trials and perturbation trials separately. Figure $3 a$ shows the two sets of VMIs plotted against each other. The individual points largely fall below the unity line (Wilcoxon signed-rank test, $n=103, p<0.0001$ ), indicating a shift toward a visually driven profile in the neurons' responses (see also histogram). Note that several canonical movement neurons based on VMI (right-most grid column) transform into visuomovement neurons in the perturbation condition, unmasking the presence of latent "visual" activity throughout the intermediate/deeper layers. The top and bottom panels of Figure $3 b$ respectively show the population average of 11 movement neurons (VMI, $>0.9$ ) as well as the activity of an example movement neuron in the two conditions.

We then looked at whether a neuron's functional classification, as defined by the VMI, has an influence on its susceptibility to the perturbation. We computed a modulation index for each neuron based on the relative change in its activity during the visual epoch on perturbation trials compared with control trials (see Materials and Methods). The modulation index is plotted against the VMI for each neuron in Figure $3 c$. The two indices were highly correlated (Pearson's correlation coefficient, 0.571; $p<0.0001$ ), indicating that neurons with a more movementrelated profile (VMI closer to +1 ) are modulated to a greater extent by disruption of fixation. These results suggest that the contribution of individual neurons to visuomotor transformations and saccade generation is not fixed but is flexibly modulated, depending on the state of the network.

\section{Fixation-related activity in the rostral SC is dissociated from visuomovement activity}

Rostral SC neurons, which fire at a tonic rate during fixation and burst for microsaccades, are suppressed during larger saccades, and there is some evidence for reciprocal inhibitory connectivity between the rostral and caudal SC (Meredith and Ramoa, 1998; Munoz and Istvan, 1998; but see also Phongphanphanee et al., 2014). We therefore considered whether the enhancement of visuomovement activity following perturbation in the caudal SC is obligatorily linked to a suppression of activity in the fixationrelated network in the rostral SC. Figure $4 a$ shows the population activity of 44 rostral SC neurons in the control and perturbation conditions. At the population level across all three animals, there was no consistent difference between the two conditions as seen from the significance bands below the activity traces (Wilcoxon signed-rank test, $p>0.05)$. However, the individual subjects' data presented a spectrum of effects (Fig. $4 b$ ). Monkey BB's rostral SC showed a dramatic and sustained suppression on perturbation trials, whereas Monkeys BL and WM showed a transient increase immediately after the perturbation before a return back to control levels. The heterogeneity of the effect can also be realized through the scatter plot of average firing rates during the delay period (Fig. 4c). Since the behavior and caudal SC profiles are largely consistent across subjects, while the rostral SC activity 
a
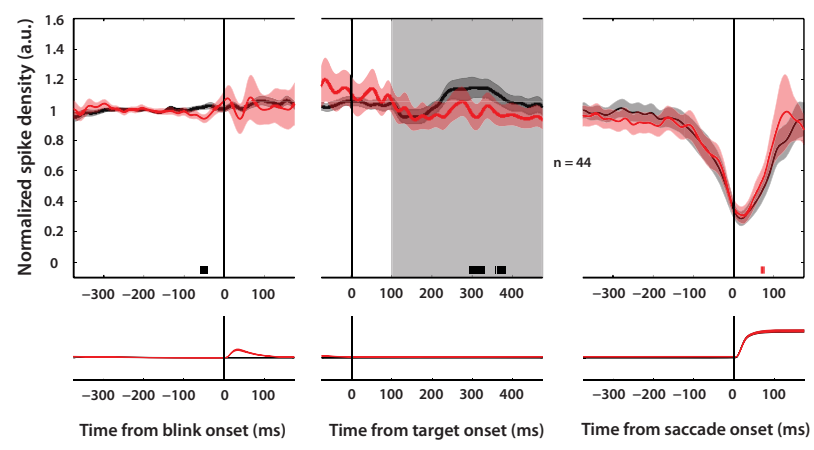

C

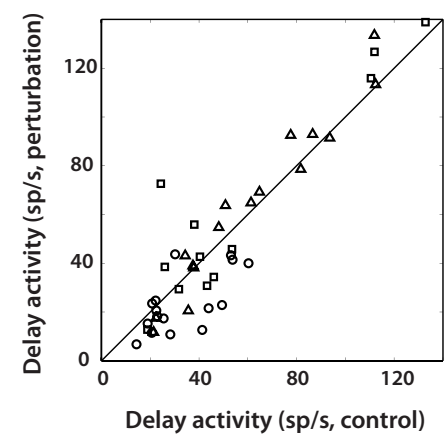

○ Monkey BB

a Monkey BL

$\Delta$ Monkey WM b
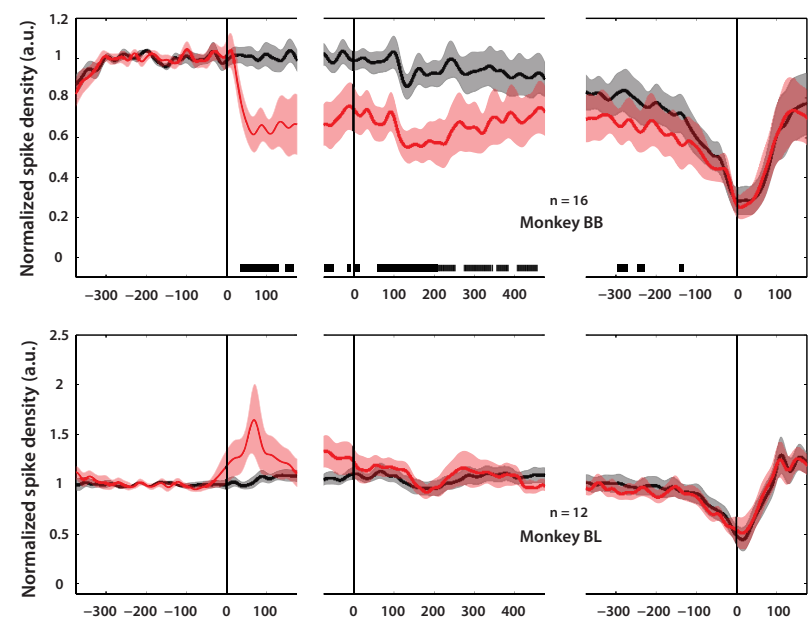
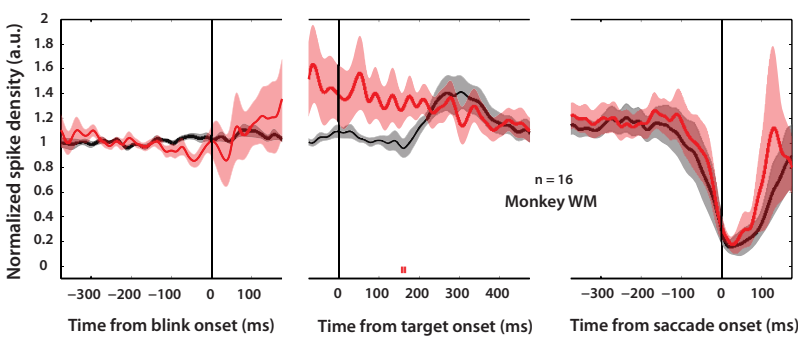

Figure 4. Population activity in the rostral SC. $\boldsymbol{a}$, Top, Population average of rostral SC neurons (normalized) in the two conditions. Colors and tick marks same as in Figure 2. The bottom row shows mean eye-position traces. $\boldsymbol{b}$, Individual subject population means of rostral SC neurons. Activity was strongly suppressed in Monkey BB whereas Monkeys BL and WM showed transient increase in rostral SC activity (not significant, Wilcoxon rank-sum test, $p>0.05$ ). $\boldsymbol{c}$, Scatter plot of the individual neurons' activities $(n=44)$ during the delay period (shaded epoch in $\boldsymbol{a}$ ) in the perturbation condition plotted against the control condition. Plot follows the scheme in Figure $2 b$.

is not, it follows that the rostral SC cannot be exclusively responsible for the shift in balance from fixation to saccade generation.

\section{Rostral SC activity is better correlated with microsaccade occurrence}

We asked whether another aspect of the animals' gaze-related behavior may help explain the heterogeneity in rostral SC and the apparent disconnect between the caudal and rostral SC populations. Since rostral SC neurons are known to play a role in the generation of tiny fixational eye movements known as microsaccades (Hafed et al., 2009), we looked at the microsaccade rate for the individual subjects as a function of time on control and perturbation trials (Fig. 5a). Monkeys BL and WM (middle and right columns) exhibited a characteristic microsaccade signature on control trials, including target-related suppression of microsaccades followed by a rebound (Winterson and Collewijn, 1976; Engbert and Kliegl, 2003). On perturbation trials, there was a pre-target reduction in microsaccade rate for these two subjects, which we attribute to the inability to detect microsaccades during the blink-related eye movement. On the contrary, we propose that the actual microsaccade rate during this period increases, a claim consistent with the increase in rostral SC activity, particularly for Monkey WM (Fig. 5b). We suggest that a blink-related reduction in brainstem omnipause neuron activity removes inhibition on the burst generators (Gandhi and Bonadonna, 2005; Schultz et al., 2010) and allows activity in upstream premotor networks (in this case, fixation activity in the rostral SC) to generate the associated movement (microsaccades) in these two subjects. On the other hand, Monkey BB, who showed a strong and sustained suppression in rostral SC activity in the perturbation condition, rarely ever made microsaccades in either condition.
Thus, microsaccades explain some of the discrepancies observed in rostral SC activity.

\section{Alternate perturbations reveal mechanism mediating fixation-saccade network balance}

We investigated whether the network-level effects observed above were specific to the perturbation method or extended to other ways of disrupting fixation. Specifically, we wanted to know whether the perturbation acts by disrupting the flow of visual information and/or the motor act of fixating, both of which are affected when the animal blinks. It is also possible that the perturbation acts as a higher-order cognitive cue informing the animal of impending target onset, thereby switching the network to a different state of preparation. To delineate these potential mechanisms, we also employed other forms of perturbations. First, we blanked out the central target during fixation at approximately the same times the blink would have occurred. This manipulation disrupts visual input to the fixation network while preserving fixation itself. Next, as a control perturbation to test the hypothesis that the cue may act in a top-down fashion, we presented a puff of air to the animal's ear during fixation. Figure $6 a, b$ shows the histogram of saccade latencies across trials and proportion of premature saccades across sessions for the targetblank and ear-puff perturbations, respectively. The target blank was similar to the blink perturbation in terms of the distribution of premature saccades - there were significantly more delayperiod errors compared with the control condition (Wilcoxon signed-rank test, $n=13$ sessions, $p<0.0001$ ). In contrast, however, the animals were not more likely to make errors following the ear puff compared with the control condition (Wilcoxon signed-rank test, $n=11$ sessions, $p>0.5$ ). Thus, it is unlikely the 
a
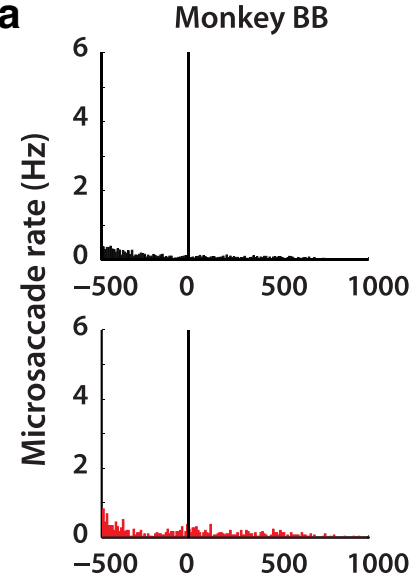

b

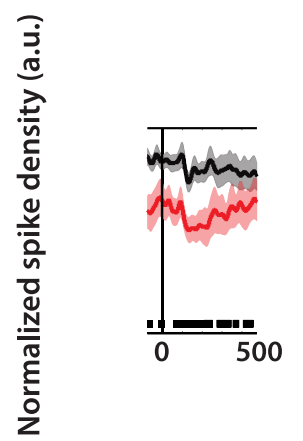

Time from target onset (ms)

Monkey BL
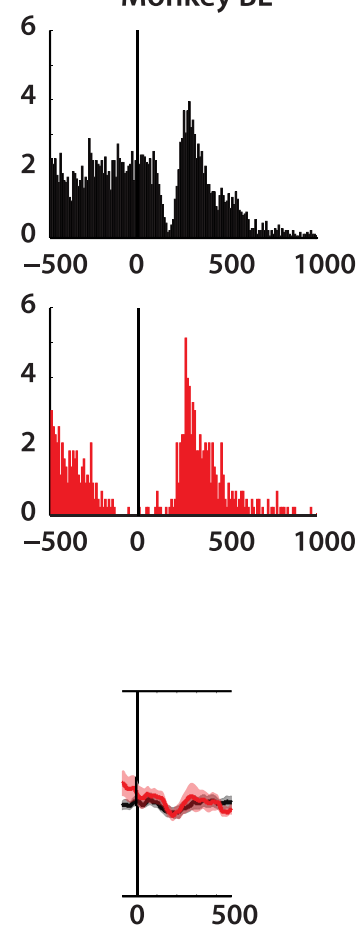
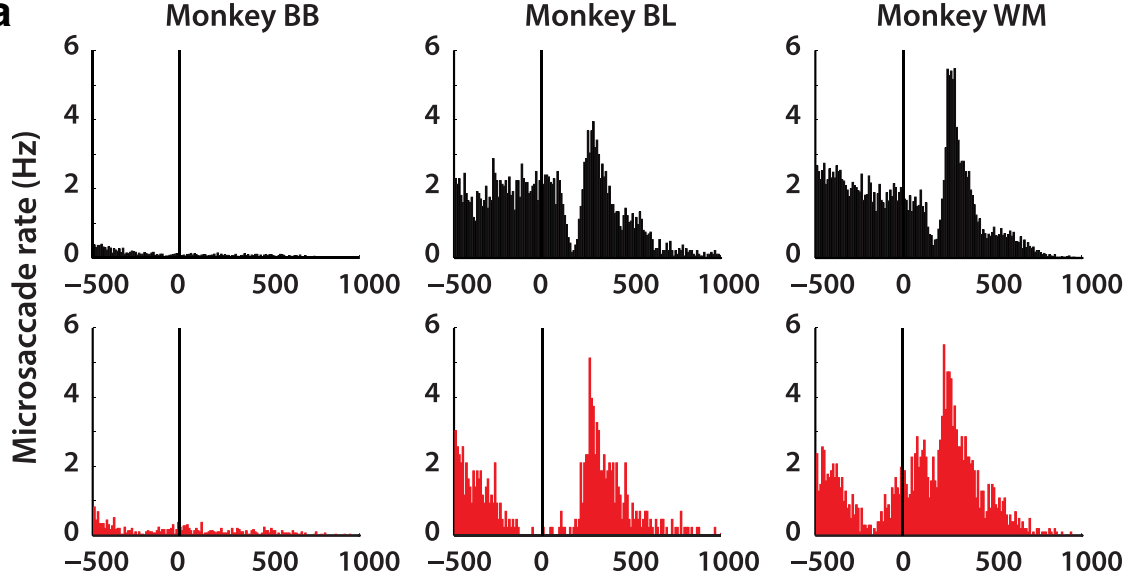

$-500$

Figure 5. Microsaccade behavior of individual animals. $\boldsymbol{a}$, Microsaccade rate as a function of time relative to target onset for each of the three monkeys in control (top row) and perturbation (middle row) trials. Monkey 1 (BB) rarely made microsaccades at any time after acquiring fixation of the central fixation target. The other two monkeys show a characteristic microsaccade rate profile with transient inhibition of microsaccades following target onset. The dramatic reduction in microsaccade occurrence in perturbation trials just before target onset is an anomaly due to the inability to detect microsaccades during the blink owing to the blink-related eye movement. $\boldsymbol{b}$, Rostral SC activity (same as Fig. $4 b$, middle column) is shown in the bottom row for comparison. It is possible that the actual microsaccade rate increases during this period (suggested by the increase in rostral $\mathrm{SC}$ activity, particularly in Monkey WM).

blink and target-blank perturbations acted exclusively as a cognitive cue to the animal.

Figure $6 c$ shows the population activity of caudal (top) and rostral SC (bottom) neurons from two animals, for these conditions, compared with the control and blink conditions for the same subset of neurons. Activity in the caudal SC was enhanced and activity in the rostral SC suppressed following the targetblank perturbation (compare blue and black traces; Wilcoxon signed-rank test, $p<0.01$ ). The suppression in the rostral SC seemed to be linked to a reduction in microsaccade rate in the one animal that made microsaccades in this condition (Monkey BL; Fig. $7 a$, middle row). There was minimal change to the network following the ear-puff perturbation (green traces), although there was a slight increase in caudal SC activity during the late component of the visual response (Fig. $6 c$, significance bars on bottom of each panel). Note that the red traces in Figure $6 c$ are from blink perturbation trials recorded for the same subset of neurons. The effects were similar to those described above for the larger population of neurons. Together, these results suggest that the perturbation must necessarily disrupt the visual and/or motor aspects of fixation to produce the network-level effects observed in this study.

Activity during premature saccades is reflective of a caudal SC-driven shift in balance

Thus far, we have focused on the subset of perturbation trials that produced regular latency saccades in the delayed saccade task.

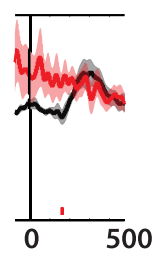

Finally, we turn to the small proportion of blink perturbation trials that resulted in saccades erroneously triggered before the GO cue (Fig. 1, gold trials). Figure 8 shows the population activity of caudal SC (top row) neurons for blink perturbation trials in which a premature saccade was produced (gold traces), compared with trials with regular latency saccades in the perturbed (red) and control (black) conditions for the same subset of neurons. Note that the difference between regular latency saccades in control and blinkperturbed trials for this subset of caudal SC neurons is similar to the effect described earlier in Figure 2-an enhancement of visuomovement activity following target onset in the perturbed condition, starting $94 \mathrm{~ms}$ after target onset (Fig. 8, top row, middle column, red vs black traces; also see red significance bar below activity traces). Caudal SC activity was even higher on premature saccade trials, and separated earlier ( $54 \mathrm{~ms}$ after target onset; see gold significance bar below activity traces) from activity in the control condition (gold vs black traces). Importantly, perturbation trials with premature saccades differentiated from trials with regular latency saccades (gold vs red traces) during the early part of the visual epoch (shaded window), with the respective activity profiles separating $92 \mathrm{~ms}$ after target onset (see arrow in significance band above activity traces).

Rostral SC activity aligned on target onset was expectedly lower during premature saccades compared with when there was no early saccade following target onset in the control or blink-perturbed conditions (Fig. 8, middle row, middle column, gold vs black and red traces). Intriguingly, compared with caudal SC activity, rostral SC activity for perturbation trials with premature saccades separated from trials with regular latency saccades much later, $\sim 200 \mathrm{~ms}$ after target onset (see arrow in middle row). This observation is consistent with the interpretation that premature movements are primarily driven by an increase in visuomovement activity in the caudal SC. It is important to highlight that visuomovement activity profiles during the visual epoch in the caudal SC (Fig. 8, top row, middle column, black, red, and gold trials) seem to fall on a continuum, suggesting that the stimulusevoked response following the perturbation is graded and may sometimes be strong enough to trigger a movement. Drawing from this observation, we wish to re-emphasize the idea that subthreshold (below the phenomenological threshold to evoke movements) neural changes can reveal the evolution of mechanisms leading to premature movements.

\section{Discussion}

We probed the functional interactions between movementgenerating neurons and movement-suppressing neurons involved in the control of gaze by perturbing fixation behaviorally, with reflex blinks, while recording neural activity in the caudal and rostral portions of the SC. We found that the perturbation 

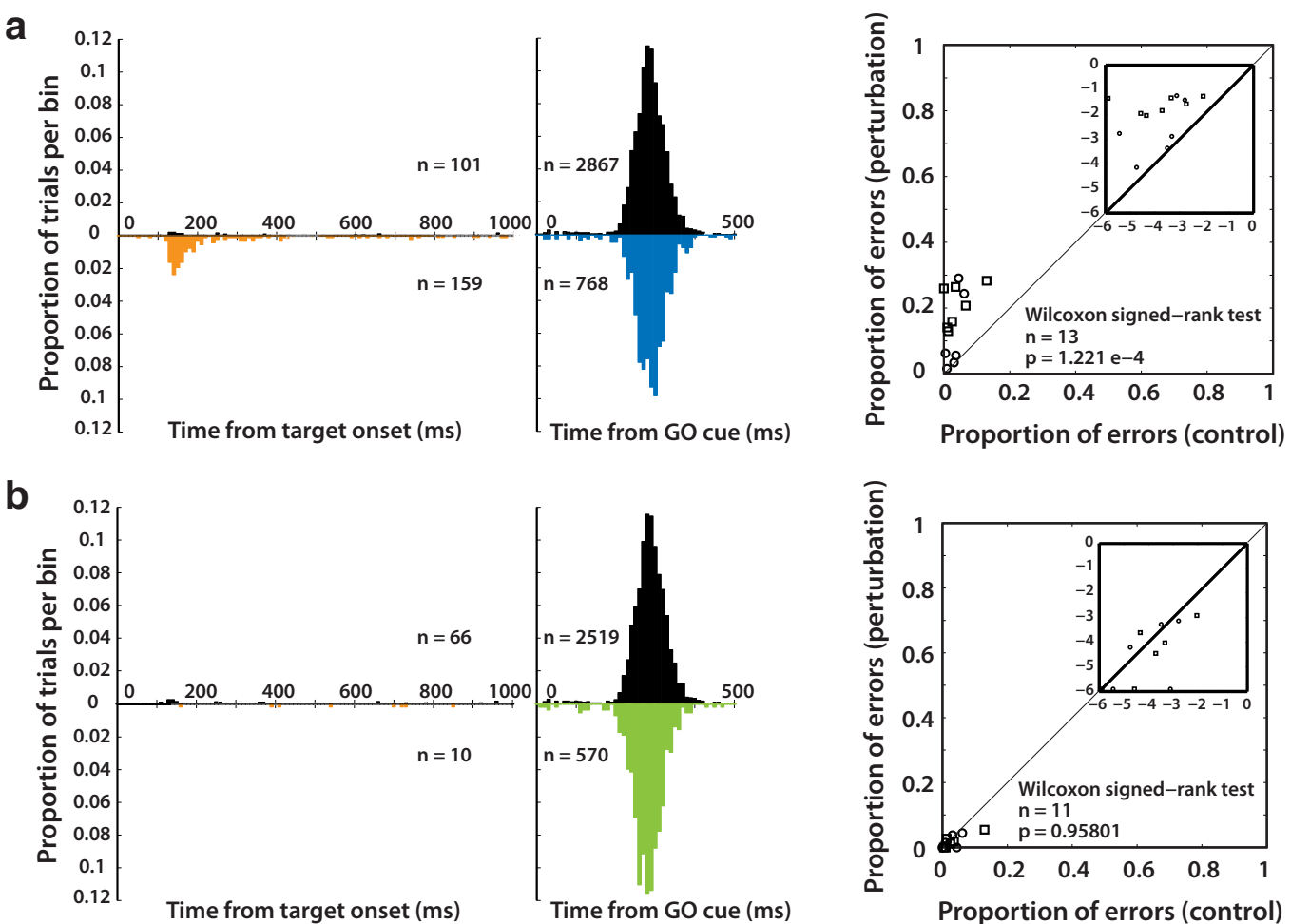

C

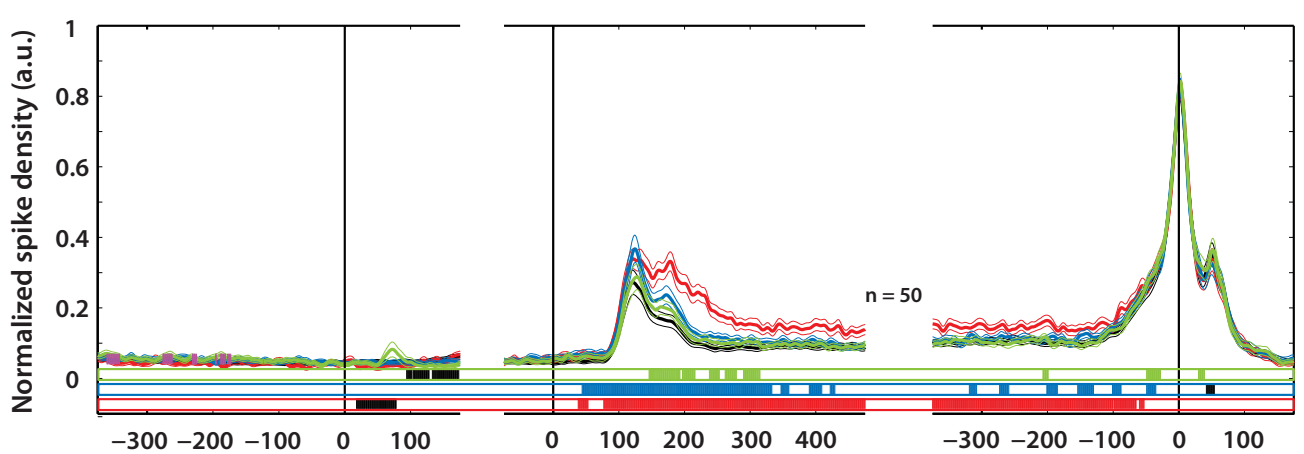

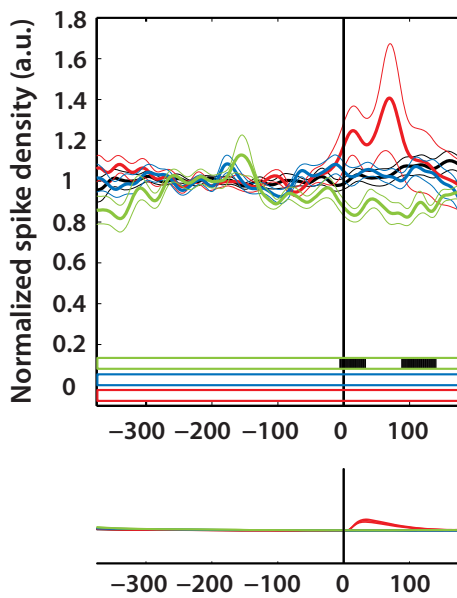

Time from blink onset (ms)

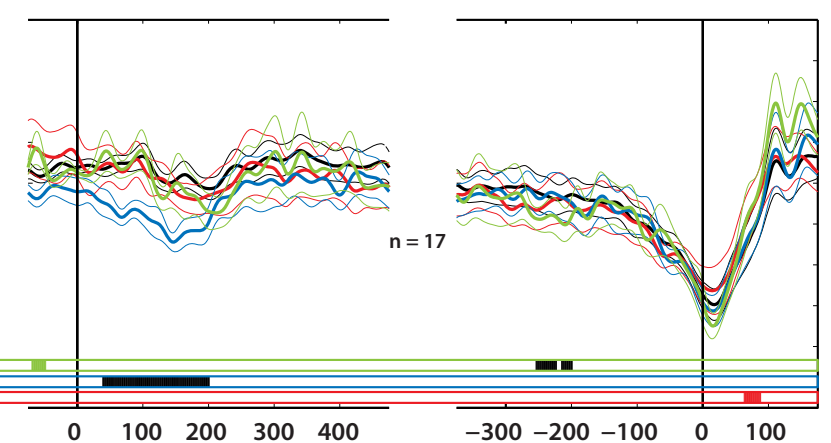

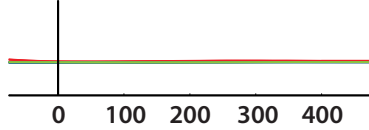

Time from target onset (ms)

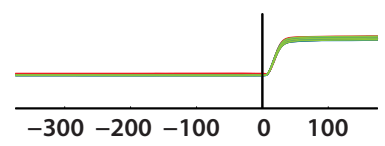

Time from saccade onset (ms)

Figure 6. Behavior and population activity for alternative perturbations. $\boldsymbol{a}$, Left, Histogram of saccade reaction times as in Figure $1 b$ for control (top) and the target-blank perturbation (bottom) trials. Right, Proportion of errors in the target-blank perturbation condition plotted against the control condition. Each point represents a session; symbols correspond to different monkeys. The unity line is on the diagonal. $\boldsymbol{b}$, Same as $\boldsymbol{a}$, but for the ear-puff perturbation. c, Population activity (mean \pm SEM) of caudal SC (top) and rostral SC (middle) neurons for each of the four conditions (control, black; blink, red; target blank, blue; ear puff, green). The activity is plotted only for the matched subset of neurons for which we had data for all four conditions. The tick marks of a particular color indicate time points at which the corresponding condition had a higher activity relative to the control condition (Wilcoxon rank-sum test, $p<0.01$ ). Specific comparisons are enclosed by thin lines of the relevant color. The bottom row shows mean eye-position traces. 
a Monkey BB

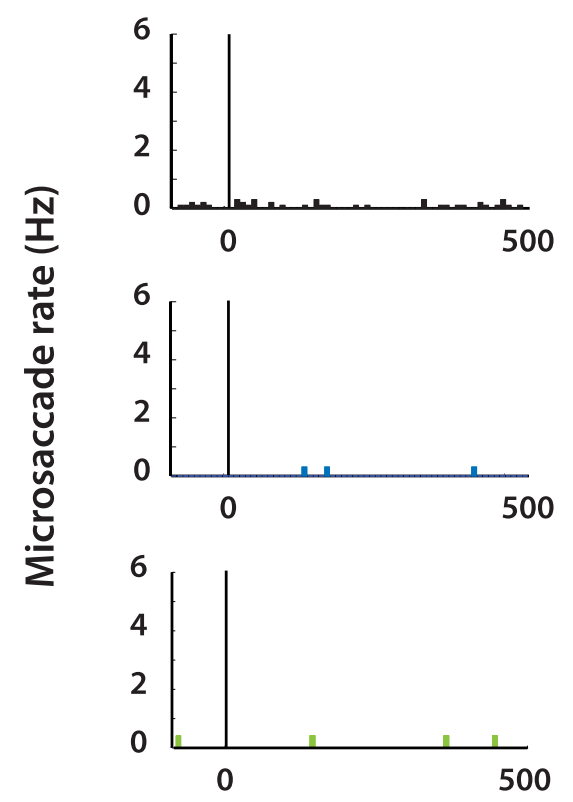

b

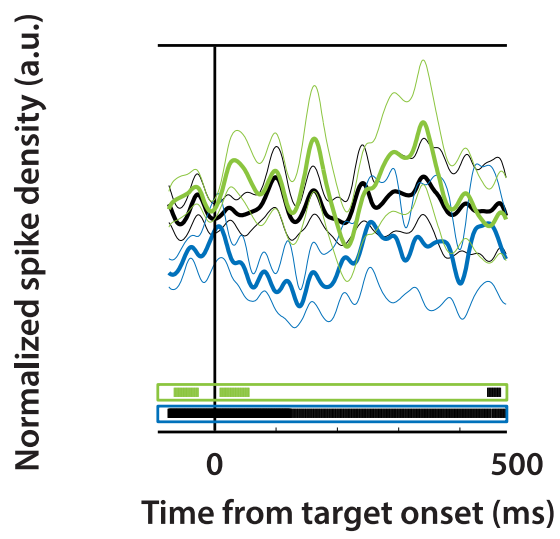

Monkey BL
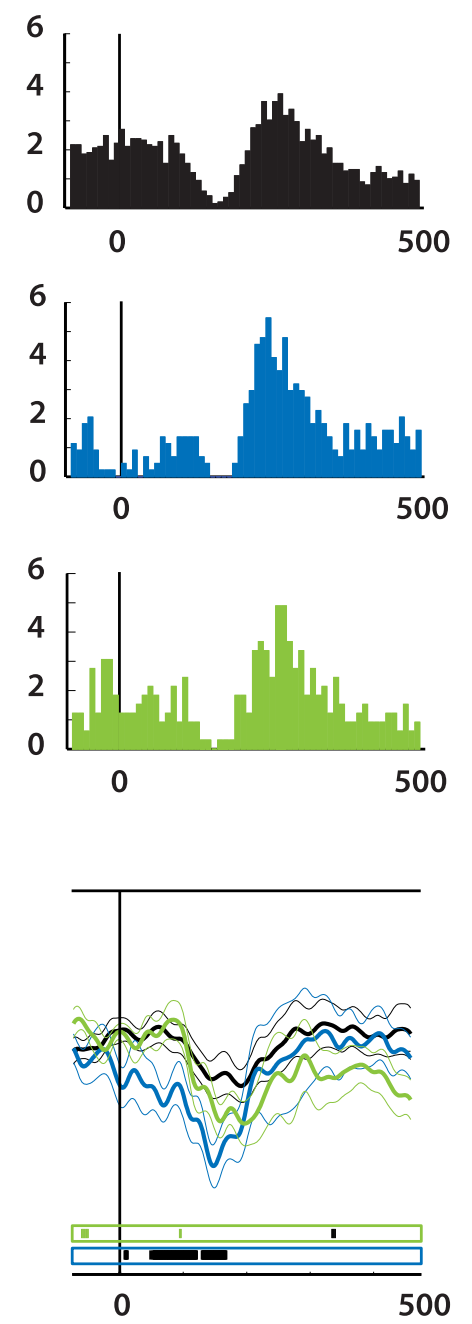

Time from target onset (ms)

Figure 7. Microsaccade behavior during alternative perturbations. $\boldsymbol{a}$, Microsaccade rate as a function of time relative to target onset for two monkeys in control (top row; data in black), target-blank (second row; data in blue), and ear-puff (third row; data in green) conditions. Just like in the blink perturbation trials (Fig. 5), Monkey BB rarely made microsaccades at any time after acquiring fixation of the central fixation target. Monkey BL showed a characteristic microsaccade rate profile with transient inhibition of microsaccades following target onset. Since there is no blink to occlude microsaccades in the target blank trials (blue histogram), the observed reduction in microsaccade occurrence just before target onset could possibly be attributed to the reduction in rostral SC activity (bottom row). $\boldsymbol{b}$, The bottom row plots rostral SC activity during the three conditions. The color configuration follows from above. The tick marks of a particular color indicate time points at which the corresponding condition had a higher activity relative to the control condition (Wilcoxon rank-sum test, $p<0.01$ ). Specific comparisons are enclosed by thin lines of the relevant color.

produced profound changes in the neural activity even when the animal's reaction time was not compromised. The perturbation increased the intensity of the visual burst in visuomovement neurons in the caudal SC and revealed novel sensorimotor properties of these saccade-generating neurons. Neurons that were more movement-like along the visuomovement continuum were more likely to be affected by the perturbation and tended to exhibit more visual-like properties. We also found that disruption of fixation had a complex effect on the rostral SC. The tonic activity of rostral SC neurons exhibited minimal changes at the population level across subjects but showed dramatic differences between them. We attributed some of these differences to the occurrence of microsaccades. We considered alternative explanations for these observa- tions and showed that other methods of perturbing fixation also had similar effects on the rostral-caudal SC network; however, it was important that the perturbation disrupted fixation either visually or by inducing an eye movement. A control perturbation with the potential to serve as a cognitive cue in advance of target onset, but that did not disrupt fixation, produced minimal effect on the network. Finally, we showed that perturbation-induced shifts are primarily driven by an increase in visuomovement activity in the caudal SC, which may sometimes trigger erroneous or premature saccades.

What do these results reveal about sensorimotor processing in the gaze-control network? In the delayed-saccade task, the monkeys were trained to suppress a saccade immediately following the onset of a target and hold their fixation until the GO cue. Under these conditions, visuomovement neurons in the SC produce an initial burst of activity locked to target onset but is typically insufficient to trigger a saccade. Visuomovement neurons in the SC project directly to the saccade burst generators in the brainstem (Rodgers et al., 2006), and an increase in their activity increases the drive to generate a saccade. Thus, the "visual response" is thought to be an embodied signature of the sensorimotor system registering the presence of the saccade target. In other words, it represents the latent intention to generate a saccade to the corresponding target. When fixation is disrupted, the target's representation is enhanced, indicating that the drive to look at the target increases. This interpretation is supported by the observation that, on occasion, the enhancement is sufficiently large to trigger a premature saccade (Fig. 8). It is important to note that the perturbation occurred tens to hundreds of milliseconds before target onset, and hence, these effects reflect persistent, long-lasting changes to the balance between the fixation and saccade-generating networkschanges that do not necessarily have a behavioral signature. However, the enhancement of the visual response may reflect internal changes, e.g., greater visual attention and/or increased target salience. Although the interpretation of a flexible visual-movement continuum is based on a wealth of studies that have found little differences between visual and saccade-related responses of visuomovement neurons, it is important to mention recent studies that have shown that a systematic transformation occurs between the visual and motor responses, from representing target location in space to representing actual movement metrics (Sadeh et al., 2015; Sajad et al., 2015). Our results suggest a lack of strong functional distinction between the visual and movement bursts, but specifically with respect to initiating the move- 


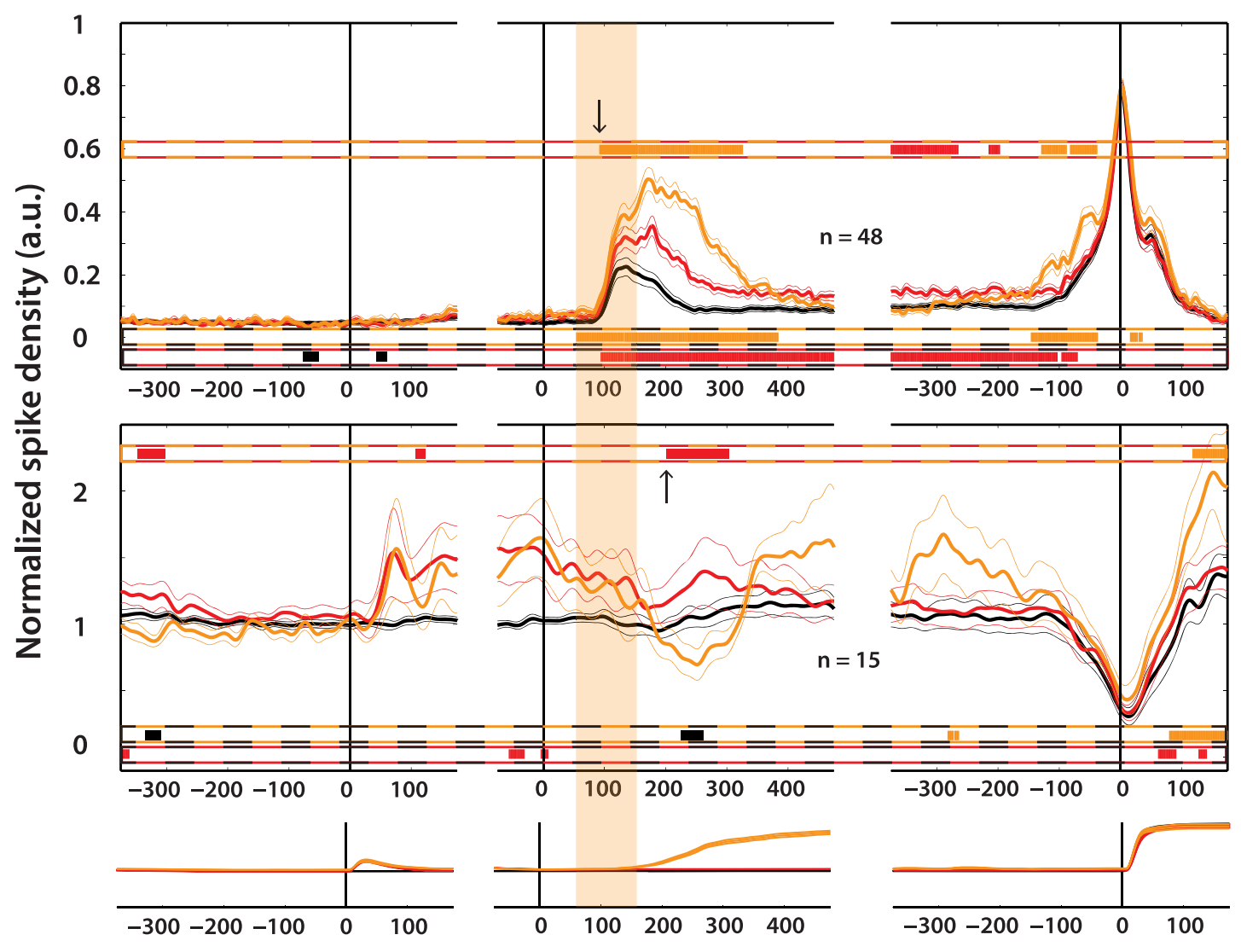

Time from blink onset (ms) Time from target onset (ms) Time from saccade onset (ms)

Figure 8. Population activity during premature saccades. Normalized population average activity (mean \pm SEM) of caudal (top row) and rostral (middle row) SC neurons for control trials (black), blink perturbation trials with regular latency saccades (red), and blink perturbation trials with early saccades (gold). Note that for the gold trials, the activity in both middle and right panels reflects saccade occurrences, but is aligned with respect to different events (target onset and saccade onset, respectively). The two sets of significance bars below the spike density profiles, enclosed by dashed black and colored lines, indicate time points at which the condition corresponding to the respective color had higher (colored bars) or lower (black bars) activity relative to the control condition. The significance bars above the spike densities, enclosed by dashed red and gold lines, indicate time points at which activity in the gold trials was higher (gold bars) or lower (red bars) compared with the red trials; the arrows indicate first times at which they significantly separated from each other (Wilcoxon rank-sum test, $p<0.01$ ). The shaded vertical rectangle indicates the early visual epoch, $50-150 \mathrm{~ms}$ after target onset. The bottom row shows the mean vectorial eye position in the three conditions.

ment. It is still possible that variation in the amplitude of the motor burst is correlated with variation in the executed saccade across trials. If so, the observed enhancement of the visual burst is consistent with the notion that the sensory response is not correlated with the actual movement.

Enhanced excitability of the saccade-generating network seems to occur through facilitation of the columnar dorsoventral pathways within the SC or through its extracollicular inputs. It does not seem to be directly related to the inability to maintain fixation as judged from the heterogeneous response of rostral SC fixation neurons. In one subject, the increased visuomovement activation was accompanied by a concomitant reduction in fixation-related activity, whereas in the others, it was associated with an increase in the firing of rostral SC neurons. Previous studies have linked such intersubject differences in the state of SC activation to idiosyncrasies in microsaccade behavior (Hafed et al., 2013). It is possible that subjects that for some reason generate few or no microsaccades have a strong functional link (or one that is apparent) between the fixation and saccade-generating networks. Other subjects that may have developed a microsaccade-based strategy for maintaining fixation may have a weaker reciprocal relationship between the two systems. In these subjects, behavioral disruption of fixation may increase activity across the SC map, and the resulting impulsive behavior may manifest as microsaccades or saccades alike. This latter interpretation is consistent with the hypothesis that microsaccades and saccades (and their associated neural substrates) form a functional continuum (Zuber et al., 1965).

It has been proposed that the rostral SC, in addition to its role in enabling fixation and fixational eye movements, is involved in maintaining gaze-related goals of the animal (Krauzlis et al., 2004). In a multistep gaze-shift task, fixation neurons in the rostral SC were shown to suppress their activity for the first step, and remained so until the end of the trial, turning on only when the complete sequence of movements was completed (Bergeron and Guitton, 2000). In at least one subject in our study, it is possible that the disruption of fixation marked the initial step, causing a sustained suppression of rostral SC activity.

We considered several alternative mechanisms leading to the results in this study. The enhancement of the visual response is similar to that observed during PFC cooling (Koval et al., 2011). The PFC has been implicated extensively in the executive control of movements (Funahashi, 2001), including saccades, and PFC deactivation or damage results in an inability to inhibit premature responses driven by the stimulus (Pierrot-Deseilligny et al., 1991). These observations together suggest that the effect reported in this study may be due to an altered balance in the PFC-SC network. Could the perturbation have served as a cognitive cue to the animal about 
impending target onset, allowing top-down biasing of the SC network balance? We have seen that a couple of different perturbations produced the observed effects in this study. However, the control perturbation of a puff of air to the ear had a weak, if discernible, effect. We suggest that the best possible interpretation given these observations is that even if the perturbation is a top-down cue, it must act through the visual-oculomotor pathway to effectively disrupt fixation and create a deficit that resembles executive-control disorders. Another possibility is that the perturbation acted as a "startle cue," and produced a startle response. Unexpected startle cues have been shown to excite the sensorimotor pathway (Kumru and Valls-Solé, 2006) and accelerate the execution of planned movements (Castellote et al., 2007). Since the perturbation was only applied on a small percentage of trials, it may have produced a startle response, resulting in the disinhibition of visuomovement neurons. However, the fact that the puff of air to the ear only had a weak effect suggests that any startle-related changes must act through the visuomotor pathway. Regardless of which of the above mechanisms was at play, the effective result of the perturbations was an altered balance between movement generation and inhibition in the gaze-control network.

Note that disruption of fixation occasionally resulted in premature eye movements (Figs. 1, 8, gold data). We propose that this mimics impulse-control disorders in which the ability to appropriately transition from fixation to saccade generation is compromised. Such deficits have been observed in neuropsychiatric disorders, such as attention-deficit hyperactivity disorder (Munoz et al., 2003) and schizophrenia (Braff and Geyer, 1990; Crawford et al., 2002), and in movement-control disorders, such as Parkinson's disease (Terao et al., 2011). Indeed, impulsivity and pathological gaze control is used as a common behavioral assay for executive dysfunction (Reuter and Kathmann, 2004). We propose that to gain a deeper understanding into the neural mechanisms causing impulsive movements, it is important to have access to internal changes that do not always manifest in overt behavior. Previous studies have attempted to study functional interactions in the gaze-control network by using causal manipulations, such as microstimulation or pharmacological inactivation. However, our understanding of what can be gained with those manipulations is limited considering the fact that it is difficult to record activity in the affected neuronal populations. Moreover, the invasive nature of those manipulations makes it difficult to apply them in human experiments. The simple, noninvasive, behavioral perturbations we used here are readily accessible for both animal and human studies. Importantly, they allow us to examine latent network processes. Such perturbation approaches may prove to be an essential supplement to standard neurophysiological experiments enabling a complete understanding of neural mechanisms underlying various behaviors.

\section{References}

Berardelli A, Cruccu G, Manfredi M, Rothwell JC, Day BL, Marsden CD (1985) The corneal reflex and the R2 component of the blink reflex. Neurology 35:797-801. CrossRef Medline

Bergeron A, Guitton D (2000) Fixation neurons in the superior colliculus encode distance between current and desired gaze positions. Nat Neurosci 3:932-939. CrossRef Medline

Braff DL, Geyer MA (1990) Sensorimotor gating and schizophrenia. Human and animal-model studies. Arch Gen Psychiatry 47:181-188. CrossRef Medline

Bryant CL, Gandhi NJ (2005) Real-time data acquisition and control system for the measurement of motor and neural data. J Neurosci Methods 142: 193-200. CrossRef Medline
Castellote JM, Kumru H, Queralt A, Valls-Solé J (2007) A startle speeds up the execution of externally guided saccades. Exp Brain Res 177:129-136. CrossRef Medline

Cohen JY, Pouget P, Heitz RP, Woodman GF, Schall JD (2009) Biophysical support for functionally distinct cell types in the frontal eye field. J Neurophysiol 101:912-916. CrossRef Medline

Crawford TJ, Bennett D, Lekwuwa G, Shaunak S, Deakin JF (2002) Cognition and the inhibitory control of saccades in schizophrenia and Parkinson's disease. Prog Brain Res 140:449-466. CrossRef Medline

Dorris MC, Paré M, Munoz DP (1997) Neuronal activity in monkey superior colliculus related to the initiation of saccadic eye movements. J Neurosci 17:8566-8579. Medline

Engbert R, Kliegl R (2003) Microsaccades uncover the orientation of covert attention. Vision Res 43:1035-1045. CrossRef Medline

Funahashi S (2001) Neuronal mechanisms of executive control by the prefrontal cortex. Neurosci Res 39:147-165. CrossRef Medline

Gandhi NJ, Bonadonna DK (2005) Temporal interactions of air-puff evoked blinks and saccadic eye movements: insights into motor preparation. J Neurophysiol 93:1718-1729. Medline

Gandhi NJ, Katnani HA (2011) Motor functions of the superior colliculus. Annu Rev Neurosci 34:205-231. CrossRef Medline

Gandhi NJ, Keller EL (1999) Comparison of saccades perturbed by stimulation of the rostral superior colliculus, the caudal superior colliculus, and the omnipause neuron region. J Neurophysiol 82:3236-3253. Medline

Goffart L, Hafed ZM, Krauzlis RJ (2012) Visual fixation as equilibrium: evidence from superior colliculus inactivation. J Neurosci 32:10627-10636. CrossRef Medline

Hafed ZM, Goffart L, Krauzlis RJ (2008) Superior colliculus inactivation causes stable offsets in eye position during tracking. J Neurosci 28: 8124-8137. CrossRef Medline

Hafed ZM, Goffart L, Krauzlis RJ (2009) A neural mechanism for microsaccade generation in the primate superior colliculus. Science 323:940-943. CrossRef Medline

Hafed ZM, Lovejoy LP, Krauzlis RJ (2013) Superior colliculus inactivation alters the relationship between covert visual attention and microsaccades. Eur J Neurosci 37:1169-1181. CrossRef Medline

Koval MJ, Lomber SG, Everling S (2011) Prefrontal cortex deactivation in macaques alters activity in the superior colliculus and impairs voluntary control of saccades. J Neurosci 31:8659-8668. CrossRef Medline

Krauzlis RJ, Liston D, Carello CD (2004) Target selection and the superior colliculus: goals, choices and hypotheses. Vision Res 44:1445-1451. CrossRef Medline

Kumru H, Valls-Solé J (2006) Excitability of the pathways mediating the startle reaction before execution of a volutary movement. Exp Brain Res 169:427-432. CrossRef Medline

Meredith MA, Ramoa AS (1998) Intrinsic circuitry of the superior colliculus: pharmacophysiological identification of horizontally oriented inhibitory interneurons. J Neurophysiol 79:1597-1602. Medline

Munoz DP, Istvan PJ (1998) Lateral inhibitory interactions in the intermediate layers of the monkey superior colliculus. J Neurophysiol 79: 1193-1209. Medline

Munoz DP, Wurtz RH (1993a) Fixation cells in monkey superior colliculus. I. Characteristics of cell discharge. J Neurophysiol 70:559-575. Medline

Munoz DP, Wurtz RH (1993b) Fixation cells in monkey superior colliculus. II. Reversible activation and deactivation. J Neurophysiol 70:576-589. Medline

Munoz DP, Waitzman DM, Wurtz RH (1996) Activity of neurons in monkey superior colliculus during interrupted saccades. J Neurophysiol 75: 2562-2580. Medline

Munoz DP, Armstrong IT, Hampton KA, Moore KD (2003) Altered control of visual fixation and saccadic eye movements in attention-deficit hyperactivity disorder. J Neurophysiol 90:503-514. CrossRef Medline

Phongphanphanee P, Marino RA, Kaneda K, Yanagawa Y, Munoz DP, Isa T (2014) Distinct local circuit properties of the superficial and intermediate layers of the rodent superior colliculus. Eur J Neurosci 40:2329-2343. CrossRef Medline

Pierrot-Deseilligny C, Rivaud S, Gaymard B, Agid Y (1991) Cortical control of reflexive visually-guided saccades. Brain 114:1473-1485. CrossRef Medline

Reuter B, Kathmann N (2004) Using saccade tasks as a tool to analyze executive dysfunctions in schizophrenia. Acta Psychol (Amst) 115:255-269. CrossRef Medline 
Robinson DA (1972) Eye movements evoked by collicular stimulation in the alert monkey. Vision Res 12:1795-1808. CrossRef Medline

Rodgers CK, Munoz DP, Scott SH, Paré M (2006) Discharge properties of monkey tectoreticular neurons. J Neurophysiol 95:3502-3511. CrossRef Medline

Rottach KG, Das VE, Wohlgemuth W, Zivotofsky AZ, Leigh RJ (1998) Properties of horizontal saccades accompanied by blinks. J Neurophysiol 79:2895-2902. Medline

Sadeh M, Sajad A, Wang H, Yan X, Crawford JD (2015) Spatial transformations between superior colliculus visual and motor response fields during headunrestrained gaze shifts. Eur J Neurosci 42:2934-2951. CrossRef Medline

Sajad A, Sadeh M, Keith GP, Yan X, Wang H, Crawford JD (2015) Visualmotor transformations within frontal eye fields during head-unrestrained gaze shifts in the monkey. Cereb Cortex 25:3932-3952. CrossRef Medline

Schultz KP, Williams CR, Busettini C (2010) Macaque pontine omnipause neurons play no direct role in the generation of eye blinks. J Neurophysiol 103:2255-2274. CrossRef Medline
Shen K, Paré M (2007) Neuronal activity in superior colliculus signals both stimulus identity and saccade goals during visual conjunction search. J Vis 7(5):15.1-13. CrossRef Medline

Sparks DL, Mays LE (1990) Signal transformations required for the generation of saccadic eye movements. Annu Rev Neurosci 13:309-336. CrossRef Medline

Terao Y, Fukuda H, Yugeta A, Hikosaka O, Nomura Y, Segawa M, Hanajima R, Tsuji S, Ugawa Y (2011) Initiation and inhibitory control of saccades with the progression of Parkinson's disease-changes in three major drives converging on the superior colliculus. Neuropsychologia 49: 1794-1806. CrossRef Medline

Winterson BJ, Collewijn H (1976) Microsaccades during finely guided visuomotor tasks. Vision Res 16:1387-1390. CrossRef Medline

Zuber BL, Stark L, Cook G (1965) Microsaccades and velocity-amplitude relationship for saccadic eye movements. Science 150:1459-1460. CrossRef Medline 